\title{
Stretchable Microfluidic Radiofrequency Antennas
}

\section{Citation}

Kubo, Masahiro, Xiaofeng Li, Choongik Kim, Michinao Hashimoto, Benjamin J. Wiley, Donhee Ham, and George M. Whitesides. 2010. "Stretchable Microfluidic Radiofrequency Antennas." Advanced Materials 22 (25) (April 22): 2749-2752. doi:10.1002/adma.200904201.

\section{Published Version}

doi:10.1002/adma.200904201

\section{Permanent link}

http://nrs.harvard.edu/urn-3:HUL.InstRepos:33490481

\section{Terms of Use}

This article was downloaded from Harvard University's DASH repository, and is made available under the terms and conditions applicable to Open Access Policy Articles, as set forth at http:// nrs.harvard.edu/urn-3:HUL.InstRepos:dash.current.terms-of-use\#OAP

\section{Share Your Story}

The Harvard community has made this article openly available.

Please share how this access benefits you. Submit a story.

Accessibility 


\title{
Stretchable Microfluidic Radio Frequency Antenna**
}

\author{
Masahiro Kubo ${ }^{1 \dagger}$, Xiaofeng $\mathrm{Li}^{2 \dagger}$, Choongik Kim ${ }^{1}$, Michinao Hashimoto ${ }^{1}$, \\ Benjamin J. Wiley ${ }^{1}$, Donhee $\mathrm{Ham}^{2}$ and George M. Whitesides ${ }^{1 *}$
}

[1] Prof. G. M. Whitesides, Dr. M. Kubo, Dr. C. Kim, Dr. M. Hashimoto, Dr. B. J. Wiley Department of Chemistry and Chemical Biology, Harvard University 12 Oxford St., Cambridge, MA, 02138 U.S.A.

[*] E-mail: gwhitesides@gmwgroup.harvard.edu

[2] Prof. D. Ham, X. Li

School of Engineering and Applied Sciences, Harvard University 29 Oxford St., Cambridge, MA, U.S.A.

$\left[{ }^{\dagger}\right]$ These two authors contributed equally to this work.

[**] The authors thank Yanyan Liu and Nan Sun at Harvard University for valuable discussions. This research was supported by a grant from DARPA award (W911NF-08C-0060) from iRobot, and NEC corporation for salary support of M. Kubo. This work was performed in part at the Center for Nanoscale Systems (CNS), which is supported by the National Science Foundation under NSF award no. ECS-0335765.

Keywords: Antenna, Stretchability, Microfluidic, PDMS, Ecoflex 
This paper describes a method for fabricating highly stretchable and robust antennas for radio-frequency signals. The antennas consist of liquid metal (eutectic gallium indium alloy, EGaIn) enclosed in elastomeric microfluidic channels. These antennas can be flexed, twisted, and stretched up to 2.2 times of their original length $l_{0}$, and exhibit little degradation $(<1 \%)$ in radiation efficiency even after being stretched to $l=1.50 l_{0}$ more than 100 times. This stretchability allows the resonance frequency of the antennas, which depends on the antenna length $l$, to be tuned from 0.738 to $1.53 \mathrm{GHz}$. The stretchable and robust antennas may be useful in reconfigurable and conformal structures, wearable sensors and large-area electronics, and other devices that must undergo large mechanical deformation.

"Stretchability" in electronics has the potential to open new opportunities, particularly in the area of large-area devices and systems, and in systems that require the device to conform to a non-planar surface, or to reconfigure structurally in use. ${ }^{[1,2]}$ Although large-area electronic devices on "flexible" substrates such as polymer or paper substrates have been demonstrated, ${ }^{[3,4]}$ flexible electronics is limited to nearly flat substrates. In contrast, stretchable electronics can cover almost arbitrarily curved surfaces and movable parts, and this may significantly enhance the comfort of the user or simplify the integration for a wide range of applications. ${ }^{[1-5]}$ Conventional electronic devices are usually made from rigid materials, which do not stretch or bend gracefully. For example, typical inorganic semiconductors fracture at tensile strain less than $1 \%$. New approaches to stretchable electronics are now being developed. In the recent significant advance, Rogers et al. ${ }^{[2,6]}$ have described stretchable integrated circuits with elongation up to $100 \%$ using wavy, thin silicon ribbons on pre-stretched elastic 
substrates. However, mechanical mismatch between the solid materials and elastomers in these approaches limits multiaxial stretchability.

Antennas offer new, attractive applications for stretchable electronics, such as reconfigurable antennas, ${ }^{[7]}$ antennas for limited and non-planar spaces, ${ }^{[8]}$ and wearable sensors. Two methods are commonly used to build antennas. The most common method for commercial applications uses sheet-metal processing; in this method, a metal sheet is punched, bent, and welded into the desired structure. A second method uses chemical etching and plating to make small patterns of metal. This method can make flexible antennas by patterning metal on a flexible substrate. Neither of these methods can produce stretchable antennas. Furthermore, neither method works when large deformation in antenna structure and thick metal sections (for high currents in high power transmission) must be combined.

Microstructured channels fabricated in elastomeric polymers and filled with liquid metal have recently been employed for stretchable interconnects ${ }^{[9]}$ and antennas. ${ }^{[10,11]}$ Besides its ability to produce stretchable antennas, this method features several advantages over existing methods employed in commercial applications: i) the process is fairly simple and scalable; ii) it does not involve etching or plating, and thus does not produce hazardous waste; iii) the method can be adopted to incorporate other stretchable 2D and 3D devices with dimensions down to tens of microns; iv) it allows the antenna to be easily integrated with other fluidic components for tuning, sensing, and signal modulation. All the previous studies, however, show either limited stretchability $(<40 \%)^{[10,11]}$ or require additional processing steps (e.g., punching holes) $)^{[9]}$ to obtain acceptable stretchability. This limitation arises from the fact that these approaches 
employed only one type of elastomer (PDMS) in the devices. PDMS itself has limited stretchability and would break if the strain exceeds $160 \%$. Furthermore, since only one type of elastomer was employed, the whole structure experienced a uniform strain when being stretched, and was likely to break at its weak points, such as the inlets and outlets of the microfluidic channels, and the interfaces between the elastic and rigid parts (e.g. external electrical connectors) of the devices, even under a strain much smaller than the maximal elongation (Figure S1B). For this reason, the devices suffered from poor mechanical durability. ${ }^{[10,11]}$

To improve the stretchability and the mechanical durability under strain, we have developed 'hybrid' structures that integrate silicone rubbers with different stiffness (Table 1) in building the microfluidic channels. We used a stiff silicone rubber (PDMS) where mechanical durability is required (e.g., around the external electrical connector) and soft elastomer (Ecoflex ${ }^{\mathrm{TM}}$, a soft, platinum-catalyzed silicone) where stretchability is necessary (e.g., around the electrically conductive lines). In an appropriate design of this type, when the conductive lines surrounded by soft elastomer are stretched by more than $100 \%$, the relatively stiff polymer around the rigid connector is not significantly strained; thus the hybrid structure, combined together with the liquid metal, provides mechanical durability under strain, and results in an extremely robust antenna structure that easily survives severe, repeated deformation.

Figure 1 shows a schematic of the stretchable antenna. As a proof of concept, we choose to fabricate a half-wave dipole antenna for its structural simplicity. The dipole antenna consists of two equal, linear branches separated by a small gap, and is fed by electrical signals at the gap via a $3 \mathrm{~mm}$ SMA connector and a coaxial cable. In this 
specific design, each antenna branch had a dimension of $32.5 \mathrm{~mm}(\mathrm{~L}) \times 3 \mathrm{~mm}(\mathrm{~W}) \times$ $200 \mu \mathrm{m}$ (thickness). The antenna length was first chosen to give a resonance frequency, which is inversely proportional to the antenna length, around $1 \mathrm{GHz}$; and the width and thickness were then determined to yield reasonably low electrical resistance $(<1 \Omega)$.

The antenna branches consist of a eutectic alloy of $75.5 \%$ Gallium and $24.5 \%$ Indium (EGaIn), and are embedded in microfluidic channels composed of PDMS and Ecoflex. We chose EGaIn primarily because it is a liquid at room temperature, and thus can be injected into the microfluidic channel without heating; it can also self-heal after deformation. ${ }^{[12]}$ Other favorable attributes of EGaIn include its low electrical resistivity $\left(29.4 \times 10^{-6} \Omega-\mathrm{cm}\right)$, high thermal conductivity, low toxicity, low vapor pressure, light weight, self-passivating oxide skin, and acceptable cost (see Table S1 in the Supporting Information for a list of metals and their properties). ${ }^{[13]}$

We fabricated the microfluidic channels using PDMS, a relatively stiff silicone rubber, and Ecoflex, a very soft platinum-catalyzed silicone. A thick $(\sim 3 \mathrm{~mm}$ in this study) PDMS slab, although not sufficiently elastic to be the sole material used in the intended applications, was well suited and used in regions where we needed a stiff material (for example, around the rigid SMA connector). ${ }^{[14]}$ Compared to PDMS, which breaks when the strain exceeds $160 \%$, the soft Ecoflex is highly stretchable, and can be elongated up to 10 times of its original length without breaking (Table 1); and thus it was used for the elastic insulating channels that held the antenna branches. Ecoflex peels away from a master in a manner similar to PDMS, so soft lithography can be used in its fabrication. ${ }^{[15]}$ It is also cured at a temperature similar to that used to cure PDMS and can easily be processed, and co-processed with PDMS. Ecoflex does not adhere well to fully 
cured PDMS; we therefore made a contact between the half-cured Ecoflex and half-cured PDMS, and cured the composite structure, to ensure good bonding at the boundary between these two types of silicone rubber. Detailed descriptions of fabricating antennas are given in the Supporting Information.

We compared the characteristics of two types of antennas: "all PDMS" structures (insulators made only of PDMS) and "PDMS/Ecoflex" structures (insulators surrounding the connector in PDMS, and the other parts in Ecoflex) under strain. We measured the three different properties of antennas to demonstrate the feasibility of a 'stretchable' antenna; i) radiation: the reflected $E M$ power, hence the efficiency of radiation, of the antenna under strain, ii) tunability: the resonance frequency of the antenna under strain, iii) reliability: the frequency response of reflected $E M$ power after repetitively stretching the antenna up to length $l=1.50 l_{0}$, where $l_{0}$ is the original length of the antenna.

The ratio between the reflected and incident $E M$ power as a function of stretch ratio $\left(l / l_{0}\right)$ was measured using a network analyzer (Figure 2$)$. If the antenna radiates efficiently, most of the incident $E M$ power is radiated into the free space, resulting in low reflected power. This value of reflected power, measured in $\left|S_{11}\right|$, is $-30 \mathrm{~dB},-20 \mathrm{~dB}$ and $10 \mathrm{~dB}$ when $99.9 \%, 99 \%$ and $90 \%$ of the input power is radiated from antenna, respectively: smaller values of $\left|S_{11}\right|$ indicate higher efficiency of radiation for an antenna. For the "PDMS/Ecoflex" structure, the unstretched antennas with original length $l_{0}$ exhibit $\left|\mathrm{S}_{11}\right|$ value of $\sim-33 \mathrm{~dB}$. As the antenna is stretched to length $l,\left|\mathrm{~S}_{11}\right|$ values varies from $\sim-30 \mathrm{~dB}\left(\right.$ at $\left.l=1.26 l_{0}\right)$ to $\sim-23 \mathrm{~dB}\left(\right.$ at $\left.l=1.59 l_{0}\right)$ to $\sim-16 \mathrm{~dB}\left(\right.$ at $\left.l=1.90 l_{0}\right)$ to $\sim-19 \mathrm{~dB}\left(\right.$ at $\left.l=2.20 l_{0}\right)$; this progression demonstrates that the "PDMS/Ecoflex" structure exhibits good radiation efficiency, even when stretched up to 2.2 times of its 
original length (or under tensile strain of $120 \%$ ). When stretched further, silicon rubbers (PDMS and/or Ecoflex) employed in the antennas lost adhesion (although the structure did not break), often causing leaks and break points of EGaIn (Figure S1A). On the other hand, for the "all PDMS" structure, unstretched antennas exhibit $\left|\mathrm{S}_{11}\right|$ value of $\sim-45 \mathrm{~dB}$, while antennas stretched by $7 \%$ in length exhibit $\left|S_{11}\right|$ value of $\sim-29 \mathrm{~dB}$. These antennas broke under tensile strain greater than $20 \%$, and were thus unreliable at even moderate strains (Figure S1B).

Figure 3 illustrates the change in the resonance frequency of the antenna with different stretch ratios. The resonant frequency of a half-wave dipole antenna can be calculated by

$$
f=\frac{143}{l} \times \frac{1}{\sqrt{\varepsilon_{\mathrm{eff}}}}
$$

where $f$ is the resonance frequency $(\mathrm{MHz}), l$ is the length of antenna $(\mathrm{m})$, and $\varepsilon_{\text {eff }}$ is the effective dielectric constant of the medium [a combination of the dielectric constants of silicone rubbers $(\varepsilon \sim 2.5)$ and air $(\varepsilon=1)$ in this study]. When the length of the antenna increases, the resonant frequency decreases accordingly. In our experiment, the resonance frequency of the antenna decreased from $1.53 \mathrm{GHz}$ to $0.738 \mathrm{GHz}$ as it was stretched from $l_{0}$ to $l=2.20 l_{0}$. As shown in Figure 5, the good agreement between our measurement and Equation (1) indicated that the antenna has worked as intended. After releasing the strain, the resonant frequency returned from $0.738 \mathrm{GHz}$ to $1.53 \mathrm{GHz}$. This reversible tuning demonstrated the robustness of our stretchable antenna.

We investigated the reliability of the antenna by repeatedly stretching it from $l_{0}$ to $l=1.50 l_{0}$. (Figure 4). Even after being stretched over 100 times, the antenna exhibited a resonance frequency nearly the same (within $1 \%$ ) as the initial measurement. Thus, the 
combination of a liquid metal antenna with a highly elastic insulating material results in an antenna structure that repeatedly returns to its original shape, even after multiple deformations, without losing its electromagnetic properties. In addition, the antennas exhibit little change (within $1 \%$ ) in their properties over 4 months of storage under ambient conditions.

In conclusion, we have developed a new method to build stretchable antennas with tunable resonance frequencies, by injecting liquid metal into a microfluidic channel in an elastomeric structural matrix. This microfluidic channel comprises two types of silicone rubber with different stiffness to significantly improve the mechanical durability and stretchability. The structure of the antenna developed in this study has three advantages over existing stretchable antennas: i) This antenna is highly stretchable, and thus has a wide tuning range. By stretching the antenna, the resonance frequency can be tuned from 0.738 to $1.53 \mathrm{GHz}$. ii) This antenna is more durable than the one fabricated in one type of silicone rubber (e.g. all PDMS). Antennas made using the hybrid PDMS/Ecoflex structure exhibit over $95 \%$ efficiency in radiation at a tensile strain of $120 \%$. iii) This antenna is more reliable to repeated cyclic strain than those comprising only PDMS. The antenna preserves its electromagnetic properties after being stretched 100 times to $l=1.50 l_{0}$.

\section{References}

[1] T. Sekitani, Y. Noguchi, K. Hata, T. Fukushima, T. Aida, T. Someya, Science 2008, $321,1468$. 
[2] D.-H. Kim, J.-H. Ahn, W.-M. Choi, H.-S. Kim, T.-H. Kim, J. Song, Y. Y. Huang, L. Zhuangjian, L. Chun and J. A. Rogers, Science 2008, 320, 507.

[3] C. Kim, Z. Wang, H.-J. Choi, Y.-G. Ha, A. Facchetti, T. J. Marks, J. Am. Chem. Soc. 2008, 10, 6867.

[4] A. C. Siegel, S. T. Phillips, M. D. Dickey, N. Lu, Z. Suo, G. M. Whitesides, $A d v$. Mater. 2009, in press.

[5] L. Gatzoulis, I. Iakovidis, IEEE Eng. Med. Biol. Mag. 2007, 26, 51.

[6] D.-H. Kim, J. Z. Song, W. M. Choi, H. S. Kim, R. H. Kim, Z. J. Liu, Y. Y. Huang, K. C. Hwang, Y. W. Zhang, J. A. Roger, Proc. Natl. Acad. Sci. U.S.A. 2008, 105, 18675.

[7] B. A. Cetiner, H. Jafarkhani, J.-Y. Qian, H. J. Yoo, A. Grau, F. De Flaviis, IEEE

Comm. Mag. 2004, 42(12), 62

[8] N. Tiercelin, P. Coquet, R. Sauleau, V. Senez, H. Fujita, J. Micromech. Microeng. 2006, 16, 2389

[9] (a) H.-J. Kim, T. Maleki, P. Wei, B. Ziaie, J. Microelectromech. Syst. 2009, 18, 138.

(b) H.-J. Kim, C. Son, B. Ziaie, Appl. Phys. Lett. 2008, 92, 011904.

[10] S. Cheng, A. Rydberg, K. Hjort, Z. Wu, Appl. Phys. Lett. 2009, 94, 144103.

[11] J.-H. So, J. Thelen, A. Qusba, G. J. Hayes, G. Lazzi, M. D. Dickey, Adv. Funct. Mater. 2009, 19, 1.

[12] M. D. Dickey, R. C. Chiechi, R. J. Larsen, E. A. Weiss, D. A. Weitz, G. M. Whitesides, Adv. Funct. Mater. 2008, 18, 1097.

[13] R. C. Chiechi, E. A. Weiss, M. D. Dickey, G. M. Whitesides, Angew. Chem. Int. Ed. 2007, 120, 148.

[14] K. M. Choi, J. A. Rogers, J. Am. Chem. Soc. 2003, 125, 4060.

[15] Y. Xia, G. M. Whitesides, Angew. Chem. Int. Ed. 1998, 37, 550. 
Table 1. Mechanical properties of PDMS and Ecoflex used as insulating material in stretchable antenna.

\begin{tabular}{lccc}
\hline & Elongation at Break $^{\mathrm{a}}(\%)$ & Shore Hardness $^{\mathrm{b}}$ & Tear Strength $^{\mathrm{c}}$ (pli) \\
\hline PMDS-184 & 160 & A-48 & 15 \\
Ecoflex-0030 & 900 & $00-30$ & 38 \\
\hline
\end{tabular}

${ }^{a}$ The strain on a sample when it breaks.

${ }^{\mathrm{b}}$ A measure of the hardness of a material, typically used for polymers, elastomers and rubbers. Materials measured in Shore 00 scale are much softer than those measured in Shore A scale.

${ }^{\mathrm{c}}$ The tensile force required to tear a pre-slit sample film of unit thickness, measured in pli or pounds per linear inch. 
Figure 1. Schematic of the stretchable antenna. The half-wave dipole antenna is made of EGaIn embedded in microfluidic channels composed of PDMS and Ecoflex. EGaIn is injected by positive pressure into the inlet, and the EGaIn-filled microfluidic channels are sealed with epoxy resin.
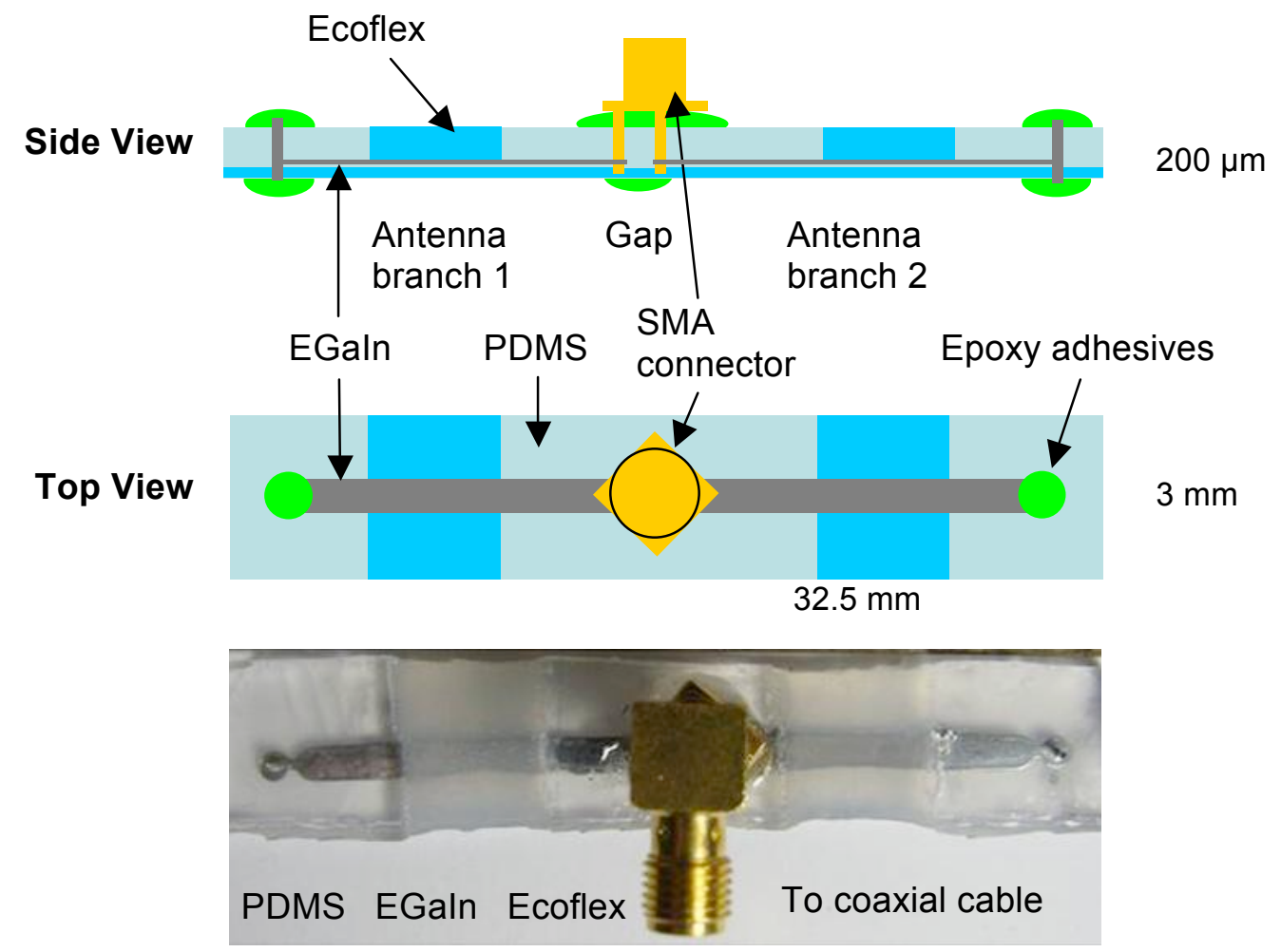
Figure 2. The reflected power $(\mathrm{dB})$ from the antenna as a function of the stretch ratio $\left(l / l_{0}\right)$. The symbols $(\diamond)$ show the reflected power of "PDMS/Ecoflex" hybrid structure while the symbols $(\diamond)$ show that of "all PDMS" structure at their resonance frequencies. Measurement under each stretch ratio was repeated five times. The "PDMS/Ecoflex" hybrid structure exhibited good radiation efficiency when stretched from $l_{0}$ up to $l=2.20$ $l_{0}$, while the "all PDMS" structure failed at a tensile strain greater than $20 \%$.

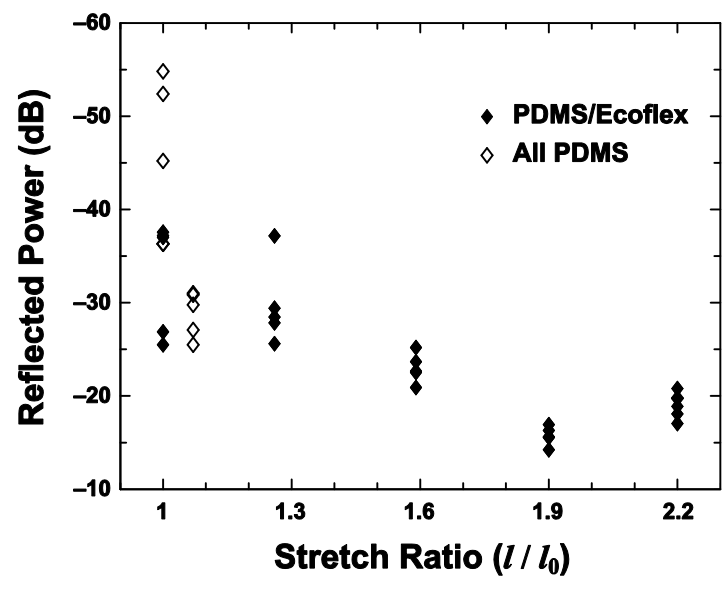


Figure 3. The resonance frequency $(\mathrm{GHz})$ of the antenna as a function of the length of antenna (in unit of $l_{0}$ ). The resonance frequency of the antenna decreased from $1.53 \mathrm{GHz}$ to $0.738 \mathrm{GHz}$ as the antenna was stretched from $l_{0}$ to $l=2.20 l_{0}$. After releasing the strain, the resonant frequency returned from $0.738 \mathrm{GHz}$ to $1.53 \mathrm{GHz}$ : the tuning is thus reversible. Measurement under each stretch ratio was repeated five times. The fitted curve was calculated using Equation (1) with $\varepsilon_{\mathrm{eff}}=2.1$.

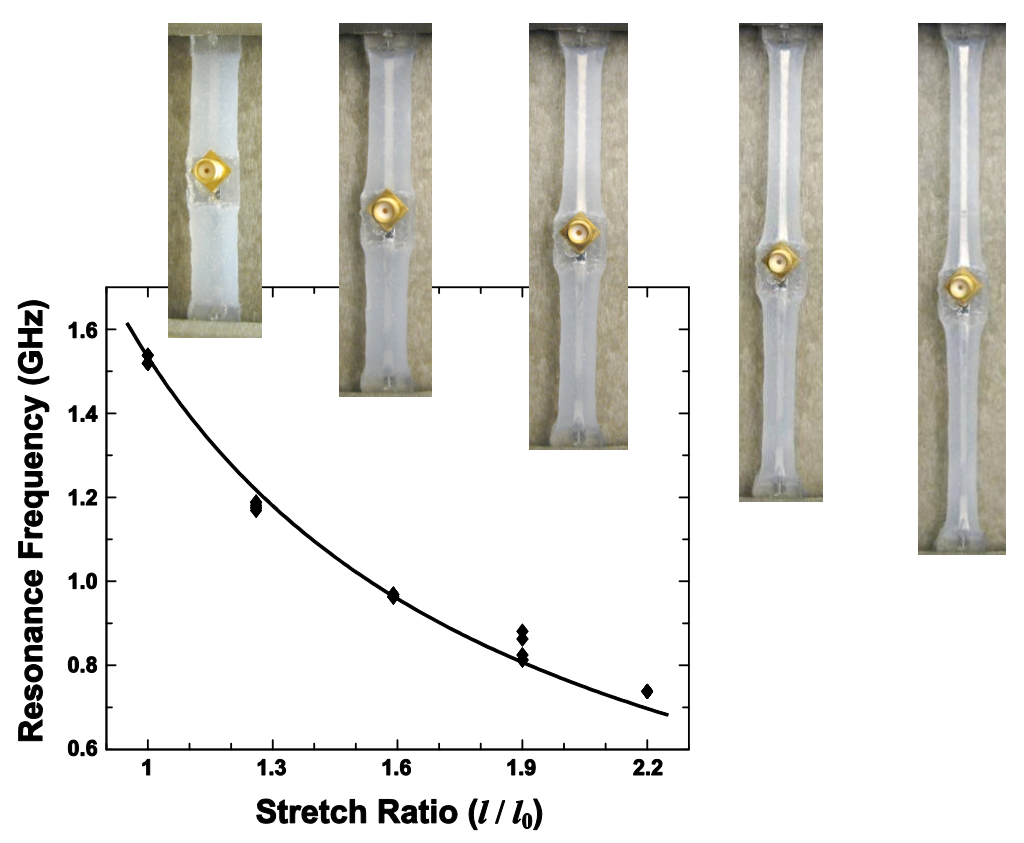


Figure 4. Frequency response of the reflected power from the antenna after repetitive stretching to $l=1.50 l_{0}$. Plots show the reflected power after the antenna had been stretched 10, 50, and 100 times. The results demonstrated that the resonance frequency and the radiation efficiency of the antenna were almost unchanged (within $1 \%$ ) after the antenna had been stretched 100 times.

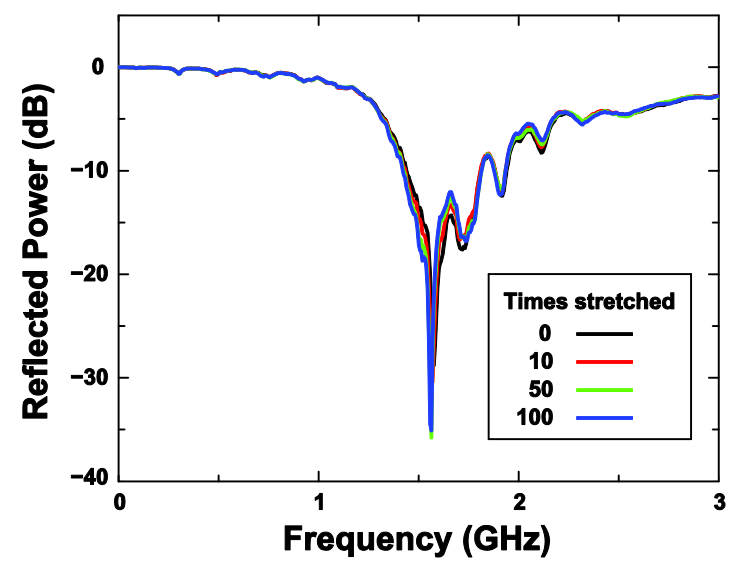




\title{
Supporting Information for
}

\section{Stretchable Microfluidic Radio Frequency Antenna}

\author{
Masahiro Kubo ${ }^{1 \dagger}$, Xiaofeng $\mathrm{Li}^{2 \dagger}$, Choongik Kim ${ }^{1}$, Michinao Hashimoto ${ }^{1}$, \\ Benjamin J. Wiley ${ }^{1}$, Donhee $\mathrm{Ham}^{2}$ and George M. Whitesides ${ }^{1 *}$
}

[1] Prof. G. M. Whitesides, Dr. M. Kubo, Dr. C. Kim, Dr. M. Hashimoto, Dr. B. J. Wiley

Department of Chemistry and Chemical Biology, Harvard University

12 Oxford St., Cambridge, MA, 02138 U.S.A.

[*] E-mail: gwhitesides@gmwgroup.harvard.edu

[2] Prof. D. Ham, X. Li

School of Engineering and Applied Sciences, Harvard University

29 Oxford St., Cambridge, MA, U.S.A. 
Table S1. Properties of Representative Metals

\begin{tabular}{|c|c|c|c|c|}
\hline Metal & $\begin{array}{c}\text { Melting } \\
\text { Point }\left({ }^{\circ} \mathrm{C}\right)\end{array}$ & $\begin{array}{c}\text { Electrical } \\
\text { Conductivity }^{\text {a, b }} \\
\left(\mathbf{1 0}^{6} \cdot \mathrm{S} / \mathrm{m}\right)\end{array}$ & $\begin{array}{c}\text { Bulk Price }^{c} \\
\text { (US\$/kg) }\end{array}$ & $\begin{array}{l}\text { Conductivity / Price } \\
(\mathrm{kS} \cdot \mathrm{kg} / \mathrm{m} \cdot \mathrm{USS})\end{array}$ \\
\hline EGaIn & 15.5 & 3.4 & 420 & 8 \\
\hline Al & 660.3 & 38.0 & 2 & 19,000 \\
\hline $\mathbf{C u}$ & 1084.6 & 59.6 & 7 & 8,800 \\
\hline Ag & 961.8 & 63.0 & 650 & 97 \\
\hline Au & 1064.2 & 45.0 & 40,000 & 1 \\
\hline $\mathbf{P t}$ & 1768.3 & 9.5 & 51,000 & 0.2 \\
\hline Hg & -38.8 & 1.0 & 16 & 63 \\
\hline Ga & 29.8 & 3.7 & 410 & 9 \\
\hline $\mathrm{Ga}^{68.5} \mathrm{In}^{21.5} \mathrm{Sn}^{10}$ & -19.0 & 3.5 & 380 & 9 \\
\hline $\mathrm{Ga}^{61} \mathrm{In}^{25} \mathrm{Sn}^{13} \mathrm{Zn}^{1}$ & 7.6 & 2.8 & 370 & 8 \\
\hline
\end{tabular}

${ }^{a}$ CRC handbook of Chemistry and Physics, CRC Press, 2009

${ }^{\mathrm{b}}$ N. B. Morley, J. Burris, L. C. Cadwallader, M. D. Nornberg, Rev. Sci. Instrum. 2008, 79, 056107.

${ }^{\mathrm{c}}$ Pure metal prices are quoted from international metal markets, including New York Mercantile Exchange (NYMEX), London Metal Exchange (LME), and warehouse in Rotterdam, Netherland. Alloy prices are calculated from pure metal prices according to the compositions of the alloys. 

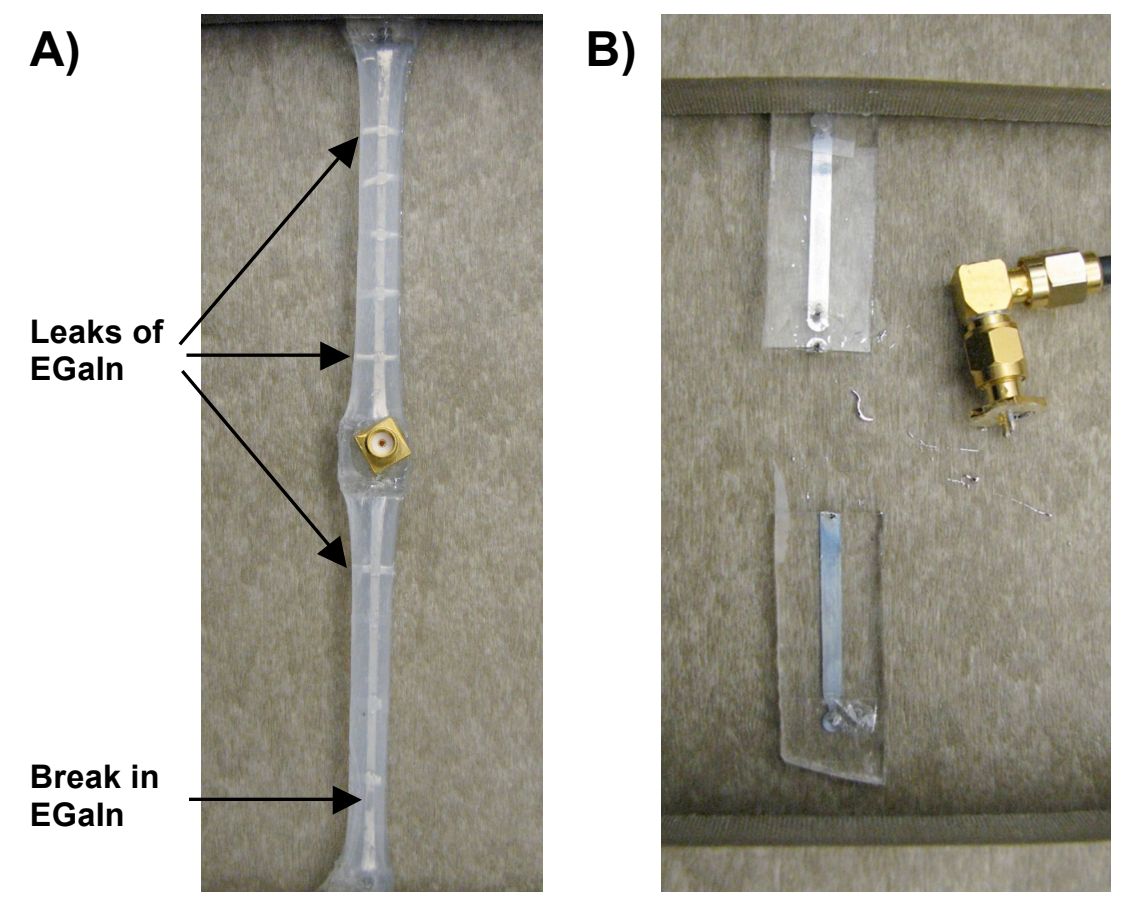

Figure S1. Photos of antennas that failed under tensile strain. A) "PDMS/Ecoflex" and B) “All PDMS” structure. 


\section{Experimental Details}

Fabrication of Stretchable Antenna (using PDMS and Ecoflex as Insulator)

Figure S2 sketches the fabrication steps of the stretchable antenna. The antenna was constructed by filling EGaIn in microfluidic channels made of insulating silicone rubbers. The microfluidic channels were formed by sticking together two half-cured silicone rubber layers. The top layer contained the microfluidic channel features that were copied from a master; the bottom layer was featureless. They together formed the microfluidic channels, and were fabricated separately.

The master for the top layer was fabricated from SU-8 photoresist (MicroChem, Inc) on a 4-inch silicon wafer (Silicone Sense Corp.). Patterns of microfluidic channels were defined by photolithography; and the resulting SU-8 master was silanized by exposing it to a vapor of $1 \mathrm{H}, 1 \mathrm{H}, 2 \mathrm{H}, 2 \mathrm{H}-$ perfluorooctyl trichlorosilane (Aldrich) overnight. On the SU-8 master, the areas that would eventually comprise Ecoflex were masked with polyimide tapes (Figure S2A). Poly(dimethylsiloxane) (PDMS) (Dow Corning Sylgard $184,10: 1)$ was poured onto this master and partially cured in an oven at $60{ }^{\circ} \mathrm{C}$ for $30 \mathrm{~min}$ (Figure S2A). To open the areas that would eventually comprise Ecoflex, the PDMS was cut by a razor blade along the edges of the polyimide tapes, which were then stripped away, removing the PDMS from these areas (Figure S2B). Freshly prepared Ecoflex (type 0030, Reynolds Advanced Materials, within 10 min after mixing the hardner and prepolymer at a ratio of 1:1) was then poured into these open areas, and partially cured at $60{ }^{\circ} \mathrm{C}$ for $10 \mathrm{~min}$. This resulted in a hybrid structure that consisted of silicone rubbers of different stiffness (Figure S2C). This top layer of the microfluidic channels was cut by a 
razor blade along the circumference of the silicon wafer, and then peeled off the master (Figure S2D).

The bottom layer of the microfluidic channels was built on a bare, featureless silicon wafer. We first covered a 4-inch silicon wafer with polyimide tapes so that the bottom layer could later be easily peeled away from the silicon. Ecoflex was poured onto the taped wafer, and partially cured at $60{ }^{\circ} \mathrm{C}$ for $10 \mathrm{~min}$ (Figure S2E). Then a $50 \mu \mathrm{m}$-thick (as measured by SEM) PDMS was spin-coated (2000 rpm for 30s) onto the Ecoflex, and partially cured at $60{ }^{\circ} \mathrm{C}$ for $30 \mathrm{~min}$ (Figure S2F).

With both layers ready, the top layer was placed on top of the bottom layer to form the microfluidic channels (Figure S2G). Good adhesion was achieved as both layers were still very sticky from the partial curing. After completely cured by heating at $60{ }^{\circ} \mathrm{C}$ for 3 $\mathrm{hr}$, the resulting structure was peeled away from the silicon wafer (Figure S2H). To create inlets and outlets for the microfluidic channels, through holes were punched by a needle (20 Gauge $=0.9 \mathrm{~mm}$ diameter $)($ Figure $\mathrm{S} 2 \mathrm{H})$, and the bottom openings of the holes was sealed by epoxy (Figure S2I). EGaIn (Aldrich) was injected by positive pressure into the inlet using a syringe (Figure S2J). The resulted EGaIn-filled microfluidic channels would act as the two branches of the dipole antenna. A $3 \mathrm{~mm}$ SMA connector (Digikey Inc.) was attached to the device by inserting its pins into the inlets/outlets of the microfluidic channels at the gap (Figure S2K); and the electrical connections formed naturally between the SMA connector and antenna branches as the EGaIn surrounded and wetted the pins of the connector. Finally, epoxy was applied to seal the microfluidic channels, and to fix the connector in place (Figure S2L). 


\section{Top Layer}

(A) Pour PDMS; half-cure

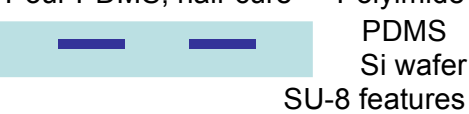

Cut the PDMS along edges of the polyimide tapes and $\checkmark$ peel away the tapes.

(B)

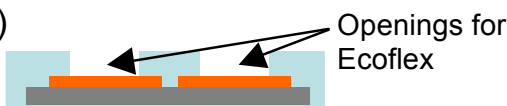

(C)

Add Ecoflex to fill the openings; half-cure

)

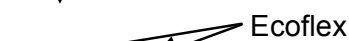

(3 $\mathrm{mm}$ thick)

(D)

Peel PDMS/Ecoflex $\checkmark$ structure from the Si mold

Bottom Layer

(E) Pour Ecoflex; half-cure Polyimide tape Ecoflex Si wafer

$(\mathrm{F})$ Spin-coat PDMS $(50 \mu \mathrm{m}$ thick); half-cure

PDMS
Attach the two half-cured

(G)

$\checkmark$ layers; cure completely

(H)

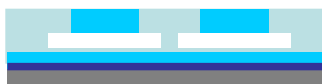

I. Peel off the structure

II. Punch through holes

(I)

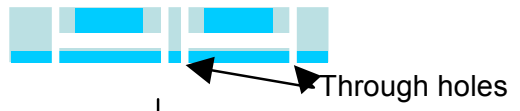

Seal bottom openings of the holes with epoxy to form inlets \& outlets

(J)

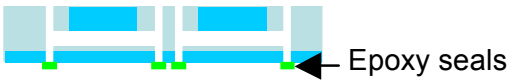
$\downarrow$ Inject EGaln to fill the channels

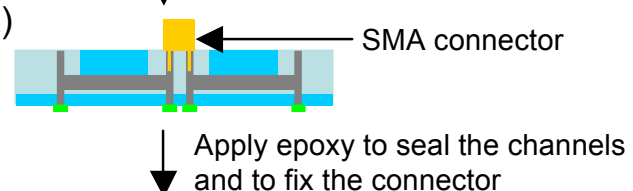

$(\mathrm{L})$

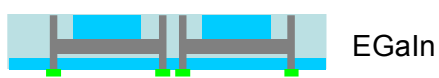

Attach the SMA connector
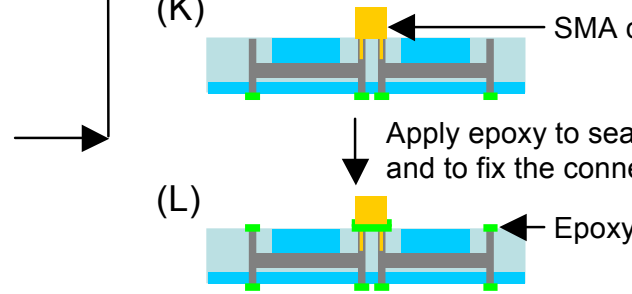

Figure S2. Steps of fabrication of the stretchable antenna. 


\section{$\underline{\text { Fabrication of Stetchable Antenna (using only PDMS as Insulator) }}$}

Antennas made in only PDMS were fabricated using a procedure similar to that we have described above. For the top layer, PDMS was poured onto the same SU-8 master and cured at $60{ }^{\circ} \mathrm{C}$ for $3 \mathrm{hr}$. The fully cured PDMS layer was cut by a razor blade along the circumference of silicon wafer, and peeled off the master. For the bottom layer, we prepared a 4-inch silicon wafer by silanizing it in a vapor of $1 \mathrm{H}, 1 \mathrm{H}, 2 \mathrm{H}, 2 \mathrm{H}$-perfluorooctyl trichlorosilane (Aldrich) overnight, so that the bottom layer could later be easily peeled away from the silicon wafer. PDMS was then spin-coated at $500 \mathrm{rpm}$ for $30 \mathrm{~s}$, resulting in a 300- $\mu$ m-thick film (as measured by SEM), and fully cured at $60{ }^{\circ} \mathrm{C}$ for $3 \mathrm{hr}$. The top layer was placed on top of the bottom layer to form the microfluidic channels. Both layers were treated with oxygen plasma for $60 \mathrm{~s}$ in order to improve the adhesion. The resulting structure was peeled away from silicon wafer. The rest steps followed the same description for the antenna using the PDMS/Ecoflex hybrid structure (Figure S2H - L).

\section{Evaluation of the Performance of the Antenna}

We measured the resonance frequency and radiation efficiency of the antenna, at its stretched and un-stretched states, using an Agilent 8358A $300 \mathrm{kHz} \sim 9 \mathrm{GHz}$ Network Analyzer (Figure S3). The antenna was put on a half-inch thick polyethylene board during evaluation, and was held in the stretched state using a pair of plastic clamps. The antenna was connected to the network analyzer directly through the rigid $50 \Omega$ SMA connector and a $50 \Omega$ coaxial cable without using a balun (balanced-unbalanced impedance transformer), because the purpose of this work is to demonstrate the stretchability and durability of our antenna, instead of characterizing a well-known dipole 
antenna. The network analyzer sent electromagnetic $(E M)$ waves at frequency $f$ to the antenna via the coaxial cable. When the $E M$ wave reached the antenna, part of its energy was radiated by the antenna into free space; a small portion was lost as heat due to ohmic resistance of the antenna; and the rest was reflected back to, and measured by, the network analyzer (Figure S3). We repeated these measurements over a range of frequencies to obtain the frequency response of reflected power from the antenna.

Half-wave dipole antenna is known to have a radiation resistance $\left(R_{\text {rad }}\right)$ close to $50 \Omega$ at its resonance frequency. ${ }^{[1,2]}$ Therefore, the incident power is maximally coupled to $E M$ radiation at the resonance frequency, resulting in a sharp dip in the frequency response of the reflected power. Such dip was indeed observed in our measurement with magnitude greater than $15 \mathrm{~dB}$ (Figure 4), meaning that more than $95 \%$ of the incident power was coupled to $E M$ radiation; and we measured the resonance frequency and radiation efficiency of the antenna from the position and magnitude of the dip, respectively.

We directly inferred the power radiated into free space from the reflected power measured by the network analyzer. For example, a reflection ratio of $-20 \mathrm{~dB}$ and $-10 \mathrm{~dB}$ corresponded to a radiation efficiency of $99 \%$ and $90 \%$, respectively. We ignored the ohmic loss inside the antenna. This is justified by the fact that the radiated power and ohmic loss are given by $I^{2} R_{\text {rad }}$ and $I^{2} R_{\text {loss }}$, respectively, for an $a c$ current $I$ flowing in the antenna, and that the radiation resistance $R_{\mathrm{rad}}(\sim 50 \Omega)$ is much greater than the ohmic resistance $R_{\text {loss, }}$, which is estimated to be less than $1 \Omega$ based on the antenna geometry and the resistivity of EGaIn. 


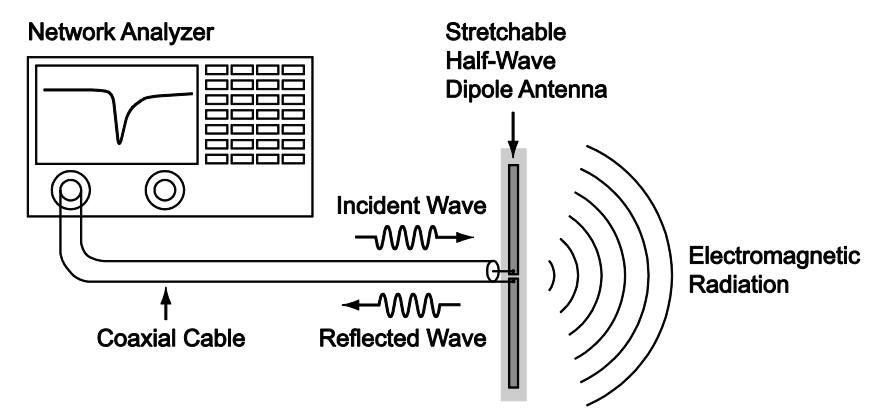

Figure S3. Measurement setup for antenna evaluation.

[1] For an ideal half-wave antenna in free space, its radiation resistance and reactance range from $50 \Omega$ to $75 \Omega$ and from $-50 \Omega$ to $50 \Omega$, respectively, around its resonance frequency $(f=0.43 \sim 0.505 c / l$, with $c$ the speed of light in free space, and $l$ the antenna length). The radiation resistance and reactance, as well as the resonance frequency, are expected to decrease when the antenna is embedded in a dielectric substrate like our stretchable antenna.

[2] Antenna Engineering Handbook ( $2^{\text {nd }}$ ed.), ed. by R. C. Johnson, H. Jasik, 1984, McGraw-Hill. 